OPEN ACCESS

Edited by:

Yue Geng,

Ministry of Agriculture and Rural

Affairs, China

Reviewed by:

Josef Velišek,

University of South Bohemia, Czechia

Jason T. Magnuson,

University of California, Riverside,

United States

*Correspondence:

Xuefeng $\mathrm{Li}$

91030@cau.edu.cn

Sen Pang

pangsen7812@cau.edu.cn

Specialty section:

This article was submitted to

Toxicology, Pollution and the

Environment,

a section of the journal

Frontiers in Environmental Science

Received: 04 January 2022

Accepted: 14 February 2022

Published: 08 March 2022

Citation:

Yu L, Li C, Zhang Y, Guo X, Cao N, Guo S, Wu S, Li X and Pang S (2022) Residue Monitoring of Propiconazole in the Rice-Crab Co-Culture Field and its

Toxicity and Bioaccumulation to

Eriocheir sinensis.

Front. Environ. Sci. 10:848348.

doi: 10.3389/fenvs.2022.848348

\section{Residue Monitoring of Propiconazole in the Rice-Crab Co-Culture Field and its Toxicity and Bioaccumulation to Eriocheir sinensis}

\author{
Lina $Y u^{1,2}$, Changsheng $L_{i}^{3}$, Yuting Zhang ${ }^{1}$, Xuanjun Guo ${ }^{1}$, Niannian Cao ${ }^{1}$, Shuxin Guo ${ }^{1}$, \\ Sijia $\mathrm{Wu}^{1}$, Xuefeng $\mathrm{Li}^{1 *}$ and Sen Pang ${ }^{1 *}$ \\ ${ }^{1}$ Department of Applied Chemistry, College of Science, China Agricultural University, Beijing, China, ${ }^{2}$ Solid Waste and Chemicals \\ Management Center, Ministry of Ecology and Environment, Beijing, China, ${ }^{3}$ College of Agronomy and Biotechnology, China \\ Agricultural University, Beijing, China
}

Rice-crab co-culture is a high-benefit eco-breeding pattern that has been extensively developed in many regions of China. However, little attention has been paid to the safety of pesticides used to control rice pests in the crab-rice co-culture system. This study monitored the actual residue levels of propiconazole in water and soil of rice-crab coculture fields and evaluated the acute toxicity, subchronic toxicity, and bioaccumulation of propiconazole to Eriocheir sinensis. We observed that the residue level of propiconazole in paddy soil was higher than that in paddy water within 42-day field monitoring. Propiconazole demonstrated a low acute toxicity $\left(96 \mathrm{~h}-\mathrm{LC}_{50}>100 \mathrm{mg} / \mathrm{L}\right)$ to $E$. sinensis and exhibited no obvious adverse impact on the growth of $E$. sinensis after exposure to $500 \mu \mathrm{g} / \mathrm{L}$, which was 10 times the actual residual concentration of propiconazole in the crab-rice co-culture field. The highest bioaccumulation of propiconazole was obtained from gills, followed by the hepatopancreas and meat. These results will contribute to the guidance of scientific utilization of pesticides in the crab-rice co-culture field.

Keywords: propiconazole, Chinese mitten crab, acute toxicity, subchronic toxicity, tissue-specific bioaccumulation

\section{INTRODUCTION}

Food shortage has become an urgent problem to be solved because of the rapid growth of population and limited water and soil resources (Bashir et al., 2020; Xu et al., 2021; Zhang et al., 2021). As one of the important staple foods, rice (Oryza sativa L.) feeds roughly half of the global population (Fageria, 2007; Liu et al., 2018). However, rice production systems have been identified as a major sector wasting available water resources, consuming up to 90 percent of irrigation water in Asia. Thus, improving the utilization of land and water resources in the rice production system is still a lacking demand (Bashir et al., 2020).

The co-culture of rice and aquatic animals has a history of more than 2,000 years in China (Wang et al., 2021). It has been considered a strategy to improve the utilization of land and water resources in paddy fields to provide both grains and aquatic animal protein products to humans (Zhang et al., 2021). The Chinese mitten crab (Eriocheir sinensis) is native to the coastal rivers and estuaries of the Yellow Sea. It is an important freshwater aquaculture species in China because of its rapid growth, strong fertility, and high nutritional values (Shen et al., 2017; Hong et al., 2019), with an annual output of over 770,000 tons (BFMA, 2020). Driven by huge economic benefits, E. sinensis has been 
developing rapidly in China in recent years and contains a variety of farming models. Rice-crab co-culture is a high-benefit ecobreeding pattern that has been extensively developed in many regions of China, especially in Liaoning Province (Yan et al., 2014). Panjin city, Liaoning Province, a representative area for cultivating crabs in paddy fields, has a rice-crab co-culture area of about 45,300 ha, accounting for $43.27 \%$ of the city's riceplanting area.

However, there are still some problems to be solved in the rice-crab co-culture system, especially the safety issues of pesticides to biological resources, for e.g., E. sinensis, when they were applied to control rice diseases, pests, and weeds. Although the crabs could eliminate some weeds and pests in the field to achieve the effect of biological pest control, there is no evidence that crabs can effectively control rice diseases. Therefore, in order to avoid rice yield reduction caused by diseases, fungicides have to be used to control rice diseases in rice-crab co-culture fields. Propiconazole was first registered in 1981 by the United States Environmental Protection Agency (EPA) in applications against a broad range of fungal diseases (Souders et al., 2019). The fungicide acts via demethylation of the C14 in ergosterol biosynthesis, an event that disrupts fungal cell wall integrity, effectively impeding further fungal growth (Johansen et al., 2007). Propiconazole is widely used in China to control rice diseases, and more than 150 formulations containing propiconazole have been registered for the control of rice diseases by the Ministry of Agriculture and Rural Affairs of China. However, propiconazole is frequently detected in the aquatic environment. Battaglin et al. detected propiconazole residues in $13 \%$ of 29 rivers in 13 states of the United States with a maximum concentration of $1.15 \mu \mathrm{g} / \mathrm{L}$ (Battaglin et al., 2010). Similarly, in Belgium, propiconazole was detected in surface water samples collected from 16 locations and ranged from 1.9 to $178.3 \mathrm{ng} / \mathrm{L}$ in effluent from waste water treatment plants (Van De Steene et al., 2010). Propiconazole has been found to have potential effects on aquatic invertebrate crabs, such as Mysidopsis bahia (28 days reproduction NOEC $=0.114 \mathrm{mg} / \mathrm{L}$ ) (European Food Safety Authority, 2017). In addition, the long half-life (DT50) of propiconazole in the environment makes it more vital to investigate the long-term influence on the related biological resources and environment. Edwards et al. reported that propiconazole showed a DT50 value of 99-116 days in agricultural practices (Edwards et al., 2016). DT50 of propiconazole in the water/sediment system was 561 days according to the European Food Safety Authority (European Food Safety Authority, 2017). Therefore, it is necessary to study whether propiconazole has potential adverse effects on E. sinensis in the rice-crab co-culture system. Although propiconazole belongs to medium bioaccumulation chemicals, indicated by the bioconcentration factor (BCF) of 180 in fish (European Food Safety Authority, 2017), whether or not propiconazole can accumulate in E. sinensis, enter, and affect human health through the food chain is still unknown.

In the present study, the residue level of propiconazole in water and soil of rice-crab co-culture fields was monitored. In addition, the acute toxicity, subchronic toxicity, and bioaccumulation of propiconazole to $E$. sinensis were

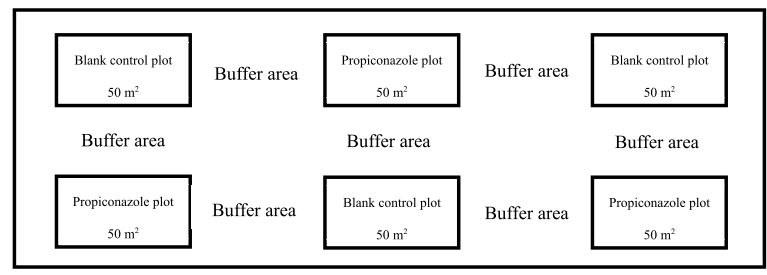

FIGURE 1 | Field experiment plot map.

evaluated. The purpose was to quantify the potential adverse effects of propiconazole on the Chinese mitten crab based on the actual residual amount of propiconazole in the rice and crab coculture fields and to provide guidance for the scientific use of propiconazole in the rice and crab co-culture system.

\section{MATERIALS AND METHODS}

\section{Chemical Material}

95\% propiconazole was purchased from Yunxin (Beijing) Agricultural Technology Co., Ltd. Stock solution of propiconazole exposure was prepared in dimethyl sulfoxide. The $25 \%$ propiconazole emulsifiable concentrate (EC) and propiconazole stock standard solutions of $100 \mathrm{mg} / \mathrm{L}$ in acetone were supplied by the College of Science, China Agricultural University. Dimethyl sulfoxide (purity 99.7\%) and Tween 80 were purchased from J\&K Scientific Ltd.

\section{Field Experiment}

One rice-crab co-culture field located in Panjin, China, was selected for this study. The experimental plots were $50 \mathrm{~m}^{2}$ and consisted of a control site and propiconazole treatment site. Each treatment was conducted in three plots, with a $50-\mathrm{m}^{2}$ plot separating each site, acting as a buffer between locations. The map of the field experiment plot is shown in Figure 1.

$25 \%$ propiconazole EC was sprayed evenly in the aforementioned experiment plots using a SeeSa SX-MD16E-2 backpack sprayer at an active ingredient dose of $45 \mathrm{~g}$ a. i/ha. The paddy water and soil samples were collected from each plot at 10 intervals up to 42 days after propiconazole application, i.e., 0 days ( $2 \mathrm{~h}), 1,3,5,7,10,14,21,30$, and 42 days. At each sampling date, at least $1 \mathrm{~kg}$ of soil (pooled sub-samples from randomly ten points) and $2000 \mathrm{ml}$ of water (pooled sub-samples from 10 random locations) were taken and stored frozen $\left(-20^{\circ} \mathrm{C}\right)$ until analysis.

\section{Acute Toxicity}

The acute toxicity of propiconazole to juvenile crabs was determined under static-renewal conditions. Crabs were exposed to a nominal concentration of $100 \mathrm{mg}$ a. i./L for $96 \mathrm{~h}$, alongside a dilution water control and solvent control $(1.0 \times$ $10^{-2} \mathrm{mg} / \mathrm{L}$ dimethyl sulfoxide $+1.0 \times 10^{-4} \mathrm{mg} / \mathrm{L}$ Tween 80$)$. Each test concentration was replicated three times. Glass fish tanks with a capacity of $60 \mathrm{~L}$ were used for the exposure operation. Each tank contained $50 \mathrm{~L}$ of exposure solutions and 10 crabs. The 
whole exposure solutions were replaced daily. Mortalities and visible abnormalities (individuals not dead but unresponsive to touching the periopods and eyestalk were considered abnormal) were recorded daily. During the acute toxicity experiment, crabs were not fed, and dead individuals were removed immediately from the tank.

\section{Subchronic Toxicity}

Juvenile crabs, after being weighed, were exposed to two sublethal concentrations $(0.05$ and $0.50 \mathrm{mg} / \mathrm{L})$ of propiconazole dissolved in water under static-renewal conditions for 28 days. In total, ten crabs in each group without replicate were exposed to glass tanks containing $50 \mathrm{~L}$ blank control, solvent control $\left(5.0 \times 10^{-5} \mathrm{mg} / \mathrm{L}\right.$ dimethyl sulfoxide+5.0 $\times 10^{-7} \mathrm{mg} / \mathrm{L}$ Tween 80$)$, and propiconazole solution. The exposure solutions were prepared as described in the acute toxicity test. The control group was fed with excess feed as a reference to ensure that adequate nutrients were available. The whole test solutions were replaced daily. The crabs were examined daily during the test period. Any mortalities or the number of molting was recorded. At the end of the test, all surviving crabs were weighed as wet weights (blotted dry).

\section{Bioaccumulation Test}

In total, sixty crabs (ca. 35 40 g) of each treatment group were exposed to 0.05 and $0.50 \mathrm{mg} / \mathrm{L}$ of propiconazole for 42 days. Solution preparation, exposure operation, and feeding patterns remained consistent with subchronic experiments. Uneaten food and feces were siphoned daily from the test chambers shortly after feeding (30 min to 1 hour).

In total, four crabs were sampled on each occasion, i.e., 0 days ( $2 \mathrm{~h}), 1,2,3,5,7,10,14,21,28,35$, and 42 days. The gill, meat, and hepatopancreas of each crab were collected and homogenized by steel balls and stored frozen $\left(-20^{\circ} \mathrm{C}\right)$ until analysis.

\section{Chemical Analysis of Propiconazole in Soil, Water, and Crab Tissues \\ Preparation of the Water Sample}

For preparation of the water sample, $10 \mathrm{ml}$ of the water sample was transferred into a $50-\mathrm{ml}$ centrifuge tube, and $10 \mathrm{ml}$ acetonitrile and $3 \mathrm{~g}$ sodium chloride were added. The solution was vortexed for $5 \mathrm{~min}$ and subsequently centrifuged at $3800 \mathrm{r} /$ $\mathrm{min}$ for $5 \mathrm{~min}$. The organic layer was transferred to a $1-\mathrm{ml} \mathrm{EP}$ tube containing $30 \mathrm{mg}$ of PSA and $100 \mathrm{mg}$ of anhydrous magnesium sulfate. After vortex and centrifugation, the supernatant was filtered through a $0.22-\mu \mathrm{m}$ membrane filter into an autosampling vial and analyzed by LC-MS/MS.

\section{Preparation of the Soil Sample}

An additional $10 \mathrm{ml}$ of water was added during sample extraction, with further preparation of samples following the same procedures as described for water sample analysis.

\section{Preparation of Crab Tissues}

For preparation of crab tissues, $1.0 \mathrm{~g}$ of thawed gills was added to a 15-ml stoppered centrifuge tube, followed by $5 \mathrm{ml}$ of acetonitrile and $3 \mathrm{~g}$ of sodium chloride. It was shaken vigorously for $5 \mathrm{~min}$ and then centrifuged at 3,800 rpm for $5 \mathrm{~min}$, and one milliliter of the supernatant was added to an EP tube containing $50 \mathrm{mg} \mathrm{C} 18$ and $100 \mathrm{mg}$ anhydrous magnesium sulfate. After vortex and centrifugation, the supernatant was tested by passing through a $0.22-\mu \mathrm{m}$ filter membrane. The samples of crab meat and hepatopancreas were processed as for crab gills.

\section{Apparatus}

All samples were detected by LC-MS/MS of Shimadzu. The ion source was electrospray ionization (ESI), and Agilent Poroshell $120 \mathrm{EC}-\mathrm{C}_{18}(2.7 \mu \mathrm{m} \times 2.1 \mathrm{~mm} \times 50 \mathrm{~mm})$ was employed at room temperature. The flow rate of acetonitrile $/ \mathrm{H}_{2} \mathrm{O}(0.1 \% \mathrm{HCOOH})=$ $70 / 30(\mathrm{~V} / \mathrm{V})$ was $0.2 \mathrm{ml} \mathrm{min}^{-1}$. MRM was selected as the detection mode in triple-quadrupole mass spectrometry. The relevant parameters and the limit of detection of this method are listed in Table 1.

\section{Data Analysis}

Statistical analyses were conducted using SPSS software (Version 20.0, SPSS Inc., Chicago, IL, United States). Data normality and homogeneity of variance were validated using the Kolmogorov-Smirnov and Levene's tests, respectively. Significant differences between the control and exposure groups were evaluated using Dunnett's one-way analysis of variance (ANOVA) with a statistical significance threshold of $p<0.05$.

\section{RESULTS}

\section{Residues of Propiconazole in Water and Soil}

As shown in Figure 2, the concentrations of propiconazole in paddy water decreased significantly during the initial 10 days of application. The peak concentration was approximately $0.005 \mathrm{mg} / \mathrm{L}$ for propiconazole, and they then remained relatively constant from 10 to 42 days.

The measured concentrations of propiconazole in soil were presented in Figure 3. The levels of soil residues of propiconazole peaks on the third day $(\sim 0.05 \mathrm{mg} / \mathrm{kg})$ after application, then reduced and fluctuated around $0.01 \mathrm{mg} / \mathrm{kg}$ during the rest of the test period.

\section{Toxicity Results}

No mortality or abnormal behaviors of juvenile crabs were observed after exposed to propiconazole $(100 \mathrm{mg} / \mathrm{L})$ for $96 \mathrm{~h}$, indicating that the acute toxicity of propiconazole to E. sinensis is low.

The growth effect on juvenile crabs by propiconazole at concentrations of 0.05 and $0.50 \mathrm{mg} / \mathrm{L}$ was also evaluated. For a 28-day exposure, no mortality or molting was observed among the test organisms. The average weight growth rates for the 0-28 days period range from 13.7 to $15.7 \%$ (Figure 4), and there was no significantly statistical difference between the treatment groups and the control ones.

\section{Bioconcentration in Crab Tissues}

The highest bioaccumulation of propiconazole was detected in gills, followed by the hepatopancreas and muscle (Figures 5-7). 
TABLE 1 | Mass spectrometric parameters.

\begin{tabular}{lcccc}
\hline Pesticide & Retention time (min) & Precursor ion & Product ion & Collision energy (eV) \\
\hline Propiconazole & 1.8 & 406 & 337 & $251^{*}$ \\
& & & -15 & -25
\end{tabular}

Asterisk is used for quantitative analysis.
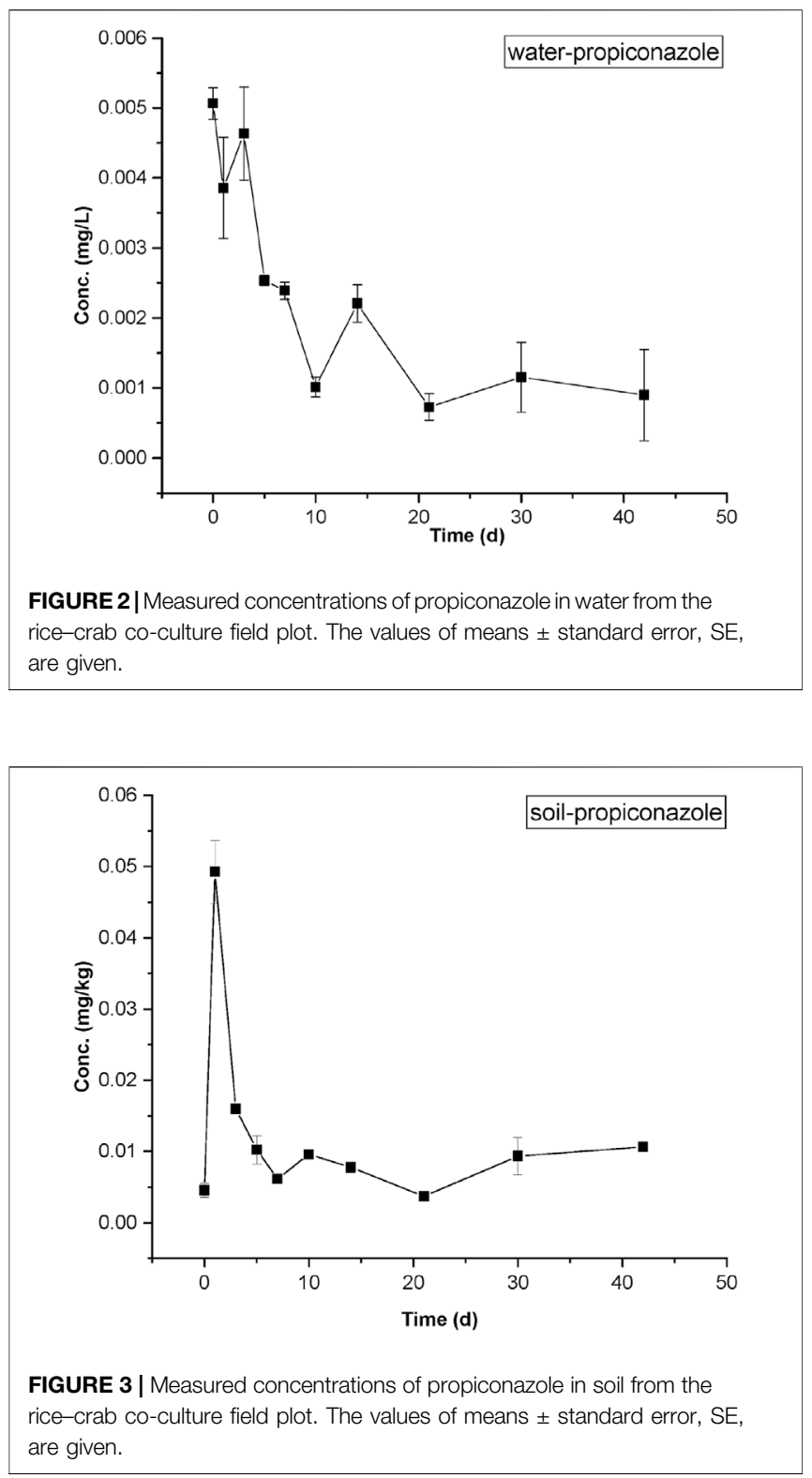

The bioconcentration of propiconazole in the gills showed a timedependent increase but did not reach a steady state during the 42day test period (Figure 5). When crabs were exposed to propiconazole for 14 days, the bioaccumulation in the muscles reached a peak and then decreased to a steady state (Figure 6). The bioconcentration of propiconazole in the hepatopancreas fluctuated during the 42-day exposure period (Figure 7).

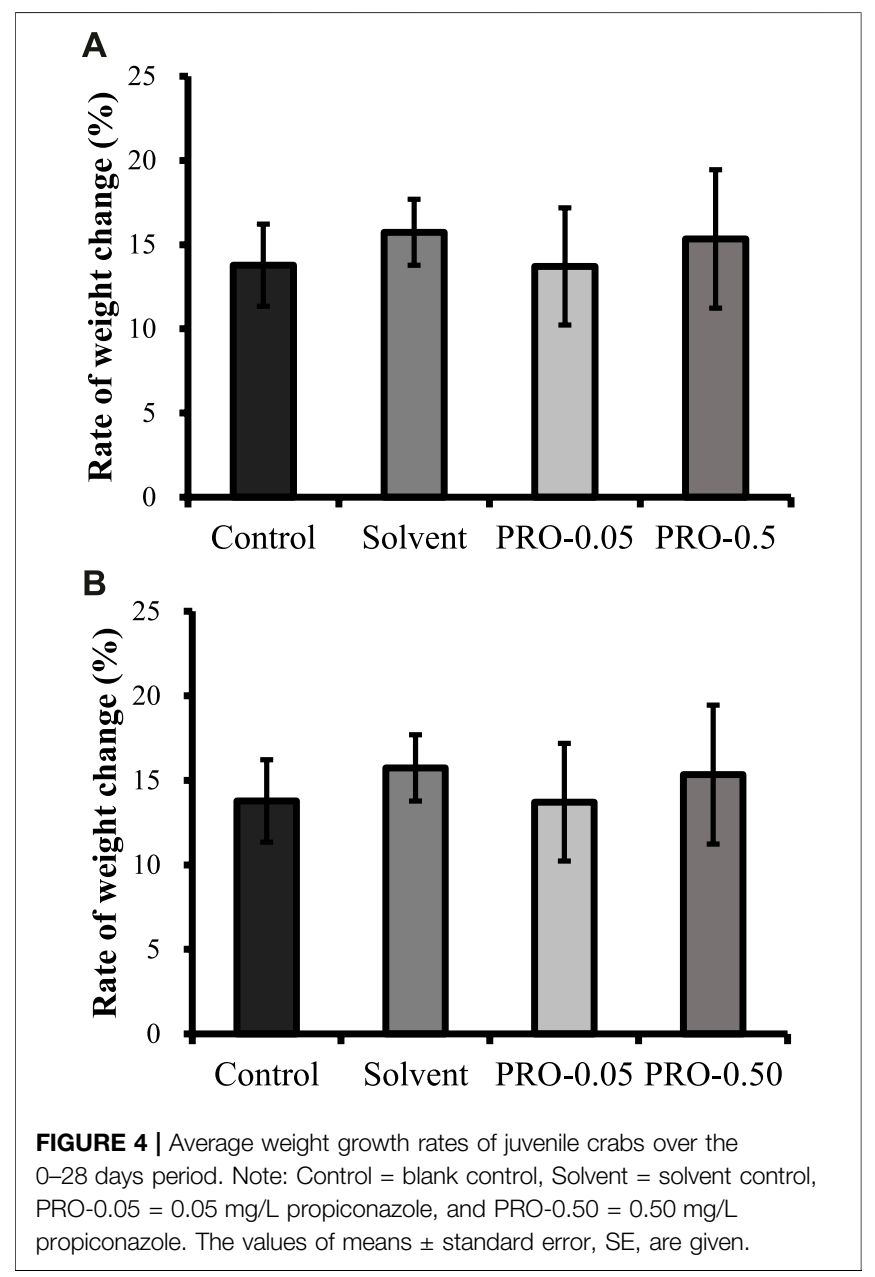

\section{DISCUSSION}

It is reported that the effective utilization rate of pesticides was only about $40 \%$ in China (Shuqin and Fang, 2018), which means that about $60 \%$ of propiconazole will enter the paddy water and soil after it is used in paddy fields. Considering the long half-life of propiconazole in water and sediments, E. sinensis raised in rice fields may be exposed to high doses of propiconazole in long terms, which may harm the health of E. sinensis and cause economic losses. Therefore, this study first monitored the dissipation of propiconazole in the rice-crab co-culture field. The dissipation dynamics of propiconazole in the rice-crab co-culture field in Panjin City, Liaoning Province, was consistent with that of triazole fungicide difenoconazole in rice fields in Guangxi and Hubei reported by Wang et al. (2012). 


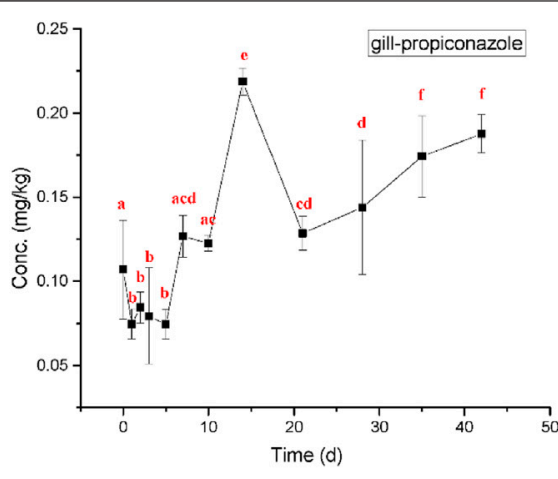

$0.05 \mathrm{mg} / \mathrm{L}$

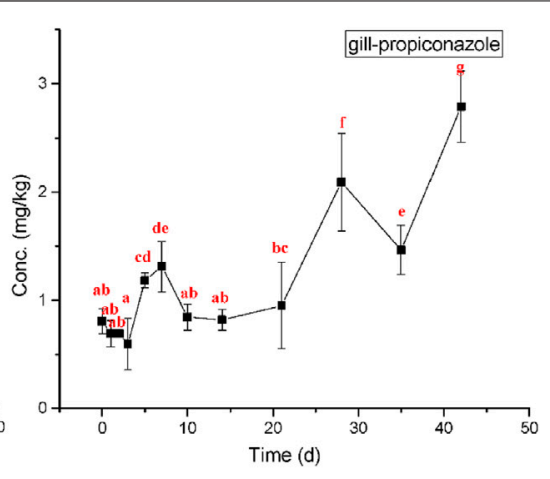

$0.5 \underline{0} \mathrm{mg} / \mathrm{L}$

FIGURE 5 | Measured concentrations of propiconazole in crab gills at concentrations of 0.05 and 0.50 mg/L over 42 days exposure. The values of means \pm standard error, SE, are given. Different lowercase letters denote significant differences $(p<0.05)$.

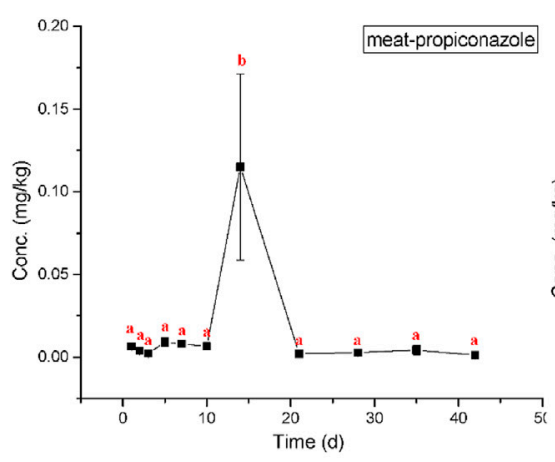

$0.05 \mathrm{mg} / \mathrm{L}$

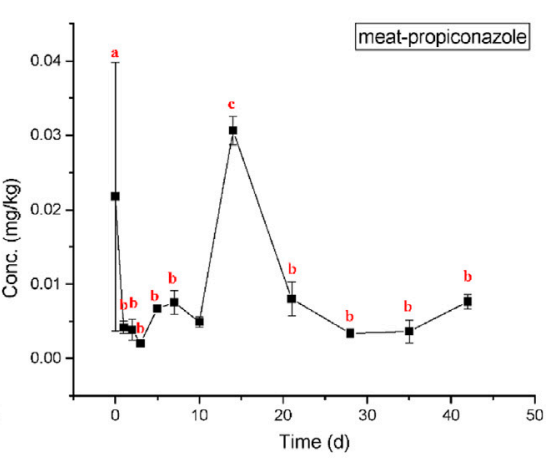

$0.5 \underline{0} \mathrm{mg} / \mathrm{L}$

FIGURE 6 | Measured concentrations of propiconazole in crab meat at concentrations of 0.05 and 0.50 mg/L over 42 days exposure. The values of means \pm standard error, SE, are given. Different lowercase letters denote significant differences $(p<0.05)$.
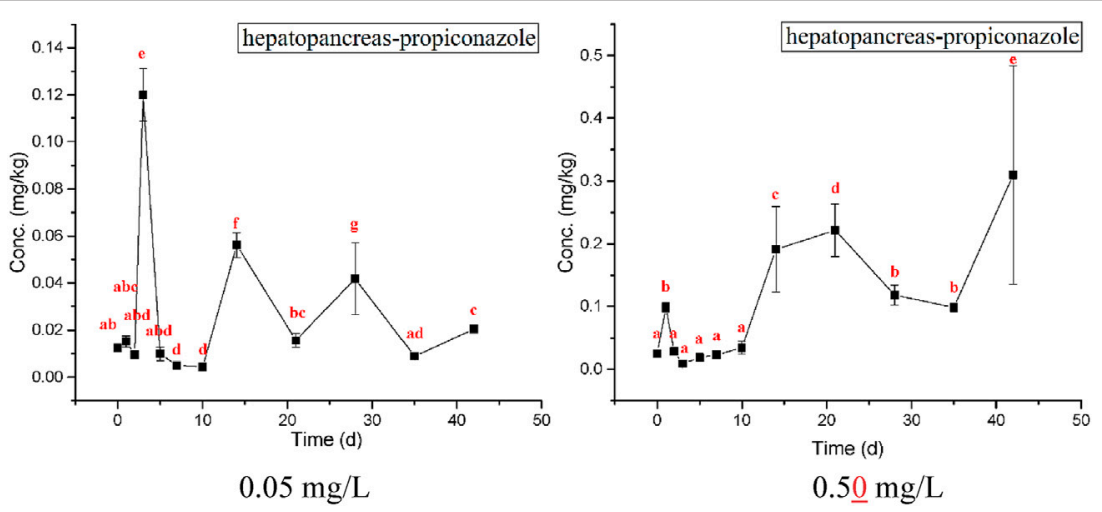

FIGURE 7 | Measured concentrations of propiconazole in the crab hepatopancreas at concentrations of 0.05 and $0.50 \mathrm{mg} / \mathrm{L}$ over 42 days exposure. The values of means \pm standard error, SE, are given. Different lowercase letters denote significant differences $(p<0.05)$.

The studies on the acute toxicity of pesticides to E. sinensis are currently mostly focused on the evaluation of the toxicity of insecticides, while there are relatively few studies on the toxicity of fungicides and herbicides. It has been reported that the organophosphorus insecticides phoxim, chlorpyrifos, triazophos, and organochlorine insecticide endosulfan were 
highly toxic to E. sinensis, while insecticides such as imidacloprid and avermectin were less toxic to E. sinensis (Li et al., 2013; Chen et al., 2014; Lu et al., 2020). Different formulations of diafenthiuron have also been found to have different toxicity to $E$. sinensis. The $96 \mathrm{~h}-\mathrm{LC}_{50}$ of diafenthiuron suspension concentrate (SC) was $42.55 \mathrm{mg} / \mathrm{L}$, while the toxicity of emulsifiable concentrate (EC) is relatively higher due to the adjuvant, $96 \mathrm{~h}-\mathrm{LC}_{50}$, was $24.66 \mathrm{mg} / \mathrm{L}$ (Gao et al., 2010). Compared with insecticides, fungicides have relatively low toxicity to $E$. sinensis. Our results demonstrated that propiconazole is a low-toxic chemical to E. sinensis. Similar results were also reported by Zhang (Zhang et al., 2014). Considering that the residual level of propiconazole in the field is unlikely to reach $100 \mathrm{mg} / \mathrm{L}$, there is no risk of acute lethality to $E$. sinensis after propiconazole is applied in the rice-crab co-cultivation field.

Since propiconazole has a long half-life in the environment, evaluating its acute toxicity alone is not enough to predict its safety to E. sinensis. It has been reported that some environmental pollutants have an impact on the growth and development of $E$. sinensis. For example, E. sinensis was exposed to chronic copper solution for 21 days, which significantly affects the weight gain of E. sinensis (Bu et al., 2022). A similar growth inhibition result of mercury chloride on E. sinensis has also been reported (Zhao et al., 2009). Our subchronic toxicity test results found that when E. sinensis was exposed to two doses of the highest residue in the field and 10 times the highest residue, propiconazole did not affect the growth of E. sinensis. However, whether propiconazole has adverse effects on the biochemical indicators of E. sinensis needs further study. Previous studies have shown that the antioxidant enzyme activities of rainbow trout were induced after long-term exposure to propiconazole ( $\mathrm{Li}$ et al., 2010). In addition, propiconazole can also induce hypopigmentation, disrupt mitochondrial bioenergetics, and can alter locomotor activity in zebrafish (Souders et al., 2019). Therefore, more biochemical and genetic studies are needed to clarify the potential harm of propiconazole to E. sinensis.

The pesticides absorbed by organisms from the surrounding environment may accumulate in the body to amplify its biological effects and may also be transmitted through the food chain, thereby adversely affecting high-level organisms. Triazole fungicides have been reported to have the risk of bioaccumulation in organisms. For instance, tebuconazole, hexaconazole, and myclobutanil have been demonstrated to accumulate in zebrafish (Andreu-Sanchez et al., 2012; Wang et al., 2015; Liu et al., 2016; Pang et al., 2020). Rainbow trout can be accumulated with a variety of triazole fungicides including bromuconazole, cyproconazole, metconazole, myclobutanil, penconazole, propiconazole, tebuconazole, tetraconazole, and triadimefon (Konwick et al., 2006). The present bioconcentration study showed that the gill and hepatopancreas of E. sinensis could be the most susceptible parts where propiconazole accumulated in. E. sinensis is one of the most popular seasonal autumn foods in China. Thus, the potential health risk induced by consuming E. sinensis should be concerned. No maximum residue levels (MRLs) of propiconazole have been applied to fish, fish products, and any other marine and freshwater food products. Therefore, MRLs which applied to products of amphibians and reptiles were referred to, i.e., $\quad 0.01 \mathrm{mg} / \mathrm{kg}$ for propiconazole [Reg (EU) 2021/155]. Measured residues of propiconazole in the gill and hepatopancreas rather than meat in the laboratory study exceeded the EU MRLs. The gill tissues of E. sinensis were inedible, but the hepatopancreas was the most favorable tissues to be consumed. In regard to health risk, the reference values of acceptable daily intake (ADI) and acute reference dose (ARfD) were established by the European Commission for propiconazole [ADI $0.04 \mathrm{mg} / \mathrm{kg}$ bw/d, ARfD $0.1 \mathrm{mg} / \mathrm{kg}$ bw; Reg (EU) 2018/1865]. Considering the limited intake of the hepatopancreas and meat during a specific season, the possibility that adverse health effects occurred to consumers due to propiconazole residues was very low.

In conclusion, it was safe for E. sinensis in the rice-crab coculture field to apply propiconazole based on normal agronomic practices. However, due to the persistence of propiconazole in the environment and its bioaccumulation in crab tissues, excessive use of propiconazole in the rice-crab co-culture field system should be avoided. In addition, the mechanism of differential bioaccumulation of propiconazole in different tissues of $E$. sinensis needed further investigations.

\section{DATA AVAILABILITY STATEMENT}

The raw data supporting the conclusion of this article will be made available by the authors, without undue reservation.

\section{AUTHOR CONTRIBUTIONS}

$\mathrm{XL}$ and SP contributed to the conception and design of the study. CL organized the database. LY, CL, YZ, XG, NC, SG, and SW performed the experiment and statistical analysis. LY wrote the first draft of the manuscript. CL and SP wrote the sections of the manuscript. All authors contributed to manuscript revision and read and approved the submitted version.

\section{ACKNOWLEDGMENTS}

The authors acknowledge technical support from Professor Yongqiang $\mathrm{Ma}$ of the Department of Applied Chemistry, College of Science, China Agriculture University. 


\section{REFERENCES}

Andreu-Sanchez, O., Paraiba, L. C., Jonsson, C. M., and Carrasco, J. M. (2012). Acute Toxicity and Bioconcentration of Fungicide Tebuconazole in Zebrafish (Danio rerio). Environ. Toxicol. 27 (2), 109-116. doi:10.1002/tox.20618

Bashir, M. A., Liu, J., Geng, Y., Wang, H., Pan, J., Zhang, D., et al. (2020). CoCulture of Rice and Aquatic Animals: An Integrated System to Achieve Production and Environmental Sustainability. J. Clean. Prod. 249, 119310. doi:10.1016/j.jclepro.2019.119310

Battaglin, W. A., Sandstrom, M. W., Kuivila, K. M., Kolpin, D. W., and Meyer, M. T. (2010). Occurrence of Azoxystrobin, Propiconazole, and Selected Other Fungicides in US Streams, 2005-2006. Water Air Soil Pollut. 218 (1-4), 307-322. doi:10.1007/s11270-010-0643-2

BFMA (2020). China Fishery Statistical Yearbook. Beijing: China Agriculture Press.

Bu, X., Song, Y., Pan, J., Wang, X., Qin, C., Jia, Y., et al. (2022). Toxicity of Chronic Copper Exposure on Chinese Mitten Crab (Eriocheir Sinensis) and Mitigation of its Adverse Impact by Myo-Inositol. Aquaculture 547, 737511. doi:10.1016/j. aquaculture.2021.737511

Chen, S., Chen, M., Song, Y., Zhou, J., and Shan, Z. (2014). Toxic Effects of Two Organic Pesticides on Eriocheir Sinensis. Environ. Sci. Technol. 37 (9), 5-14. doi:10.3969/j.issn.1003-6504.2014.09.002

Edwards, P. G., Murphy, T. M., and Lydy, M. J. (2016). Fate and Transport of Agriculturally Applied Fungicidal Compounds, Azoxystrobin and Propiconazole. Chemosphere 146, 450-457. doi:10.1016/j.chemosphere.2015.11.116

European Food Safety Authority (2017). Conclusion on the Peer Review of the Pesticide Risk Assessment of the Active Substance Propiconazole. EFSA J. 15 (7), 4887. doi:10.2903/j.efsa.2017.4887

Fageria, N. K. (2007). Yield Physiology of Rice. J. Plant. Nutr. 30 (6), 843-879. doi:10.1080/15226510701374831

Gao, Y. N., Shan, Z. J., and Cheng, Y. (2010). Risk Assessment of Diafenthiuron to Three Species of Aquatic Organisms. J. Ecol. Rural Environ. 26 (5), 487-491. doi:10.3969/j.issn.1673-4831.2010.05.016

Hong, Y. H., Huang, Y., Yan, G. W., Pan, C., and Zhang, J. L. (2019). Antioxidative Status, Immunological Responses, and Heat Shock Protein Expression in Hepatopancreas of Chinese Mitten Crab, Eriocheir Sinensis under the Exposure of Glyphosate. Fish Shellfish Immunol. 86, 840-845. doi:10.1016/j. fsi.2018.12.020

Johansen, N. S., Moen, L. H., and Egaas, E. (2007). Sterol Demethylation Inhibitor Fungicides as Disruptors of Insect Development and Inducers of Glutathione S-Transferase Activities in Mamestra Brassicae. Comp. Biochem. Physiol. C Toxicol. Pharmacol. 145 (3), 473-483. doi:10.1016/j.cbpc.2007.02.004

Konwick, B. J., Garrison, A. W., Avants, J. K., and Fisk, A. T. (2006). Bioaccumulation and Biotransformation of Chiral Triazole Fungicides in Rainbow trout (Oncorhynchus mykiss). Aquat. Toxicol. 80 (4), 372-381. doi:10.1016/j.aquatox.2006.10.003

Li, Z. H., Zlabek, V., Grabic, R., Li, P., Machova, J., Velisek, J., et al. (2010). Effects of Exposure to Sublethal Propiconazole on Intestine-Related Biochemical Responses in Rainbow trout, Oncorhynchus mykiss. Chem. Biol. Interact 185 (3), 241-246. doi:10.1016/j.cbi.2010.02.040

Li, H., Song, W. H., Li, W. K., Fu, L. J., Yao, F. X., Hu, Z. Y., et al. (2013). Acute Toxicity of Three Pesticides to Juvenile Chinese Mitten Crab (Eriocheir Sinensis). Chin. J. Fish. 26 (6), 44-47. doi:10.3969/j.issn.1005-3832.2013.06.010

Liu, N., Dong, F., Xu, J., Liu, X., and Zheng, Y. (2016). Chiral Bioaccumulation Behavior of Tebuconazole in the Zebrafish (Danio rerio). Ecotoxicol. Environ. Saf. 126, 78-84. doi:10.1016/j.ecoenv.2015.12.007

Liu, J., Liu, H. B., Liu, R., Mostofa, A., Zhai, L. M., Lu, H. M., et al. (2018). "Water Quality in Irrigated Paddy Systems," in Irrigation in Agroecosystems. Editor G. Ondrasek (London, United Kingdom: Intech Open).

Lu, J., Zhang, J., Wang, P., Qing, H., and Zhou, G. (2020). Toxicity and Safety Evaluation of Two Pesticides against Red Swamp Crayfish Procambarus clarkii and Chinese Mitten Crab Eriocheir Sinensis. Fish. Sci. 39 (6), 908-914. doi:10. 16378/j.cnki.1003-1111.2020.06.016

Pang, S., Guo, M., Zhang, X., Yu, L., Zhang, Z., Huang, L., et al. (2020). Myclobutanil Developmental Toxicity, Bioconcentration and Sex Specific
Response in Cholesterol in Zebrafish (Denio Rerio). Chemosphere 242, 125209. doi:10.1016/j.chemosphere.2019.125209

Shen, H. S., Zang, Y., Song, K., Ma, Y. C., Dai, T. H., and Serwadda, A. (2017). A Meta-Transcriptomics Survey Reveals Changes in the Microbiota of the Chinese Mitten Crab Eriocheir Sinensis Infected with Hepatopancreatic Necrosis Disease. Front. Microbiol. 8, e732. doi:10.3389/fmicb.2017. 00732

Shuqin, J., and Fang, Z. (2018). Zero Growth of Chemical Fertilizer and Pesticide Use: China's Objectives, Progress and Challenges. J. Resour. Ecol. 9, 50-58. doi:10.5814/j.issn.1674-764x.2018.01.006

Souders, C. L., 2nd, Xavier, P., Perez-Rodriguez, V., Ector, N., Zhang, J. L., and Martyniuk, C. J. (2019). Sub-lethal Effects of the Triazole Fungicide Propiconazole on Zebrafish (Danio rerio) Development, Oxidative Respiration, and Larval Locomotor Activity. Neurotoxicol. Teratol. 74, 106809. doi:10.1016/j.ntt.2019.106809

Van De Steene, J. C., Stove, C. P., and Lambert, W. E. (2010). A Field Study on 8 Pharmaceuticals and 1 Pesticide in Belgium: Removal Rates in Waste Water Treatment Plants and Occurrence in Surface Water. Sci. Total Environ. 408 (16), 3448-3453. doi:10.1016/j.scitotenv.2010.04.037

Wang, K., Wu, J. X., and Zhang, H. Y. (2012). Dissipation of Difenoconazole in rice, Paddy Soil, and Paddy Water under Field Conditions. Ecotoxicol. Environ. Saf. 86, 111-115. doi:10.1016/j.ecoenv.2012.08.026

Wang, Y., Xu, L., Li, D., Teng, M., Zhang, R., Zhou, Z., et al. (2015). Enantioselective Bioaccumulation of Hexaconazole and its Toxic Effects in Adult Zebrafish (Danio rerio). Chemosphere 138, 798-805. doi:10.1016/j. chemosphere.2015.08.015

Wang, Q., Yu, K., Ni, X., and Zhang, H. (2021). Development Process and Technical Trend of Integrated rice-fishing Cultivation. China Rice 27 (4), 88-91. doi:10.3969/j.issn.1006-8082.2021.04.018

Xu, Q., Liu, T., Guo, H., Dou, Z., Gao, H., and Zhang, H. (2021). Conversion from rice-wheat Rotation to rice-crayfish Coculture Increases Net Ecosystem Service Values in Hung-Tse Lake Area, east China. J. Clean. Prod. 319, 128883. doi:10. 1016/j.jclepro.2021.128883

Yan, Y., Liu, M. D., Yang, D., Zhang, W., An, H., Wang, Y. J., et al. (2014). Effect of Different Rice-Crab Coculture Modes on Soil Carbohydrates. J. Integr. Agric. 13 (3), 641-647. doi:10.1016/S2095-3119(13)60722-4

Zhang, G., Zhou, J., Jiang, J., Kong, D., Tian, F., Cheng, Y., et al. (2014). Impact of Propiconazole on Aquatic Organisms in Pond Water Adjacent to Paddy Field. Pestic. Sci. Admin 35 (4), 36-40. doi:10.3969/j.issn.10025480.2014.04.008

Zhang, Y., Chen, M., Zhao, Y. Y., Zhang, A. Y., Peng, D. H., Lu, F., et al. (2021). Destruction of the Soil Microbial Ecological Environment Caused by the Overutilization of the rice-crayfish Co-cropping Pattern. Sci. Total Environ. 788, 147794. doi:10.1016/j.scitotenv.2021.147794

Zhao, Y., Wang, X., Yu, Y., and Wu, J. (2009). Effect of Mercury Chloride for Survival, Growth and Molt of Juveniles of the Chinses Mitten Crab Eriocheir Sinensis. Acta Sci. Natural. U Nankaien. 2009 (3), 53-57.

Conflict of Interest: The authors declare that the research was conducted in the absence of any commercial or financial relationships that could be construed as a potential conflict of interest.

Publisher's Note: All claims expressed in this article are solely those of the authors and do not necessarily represent those of their affiliated organizations, or those of the publisher, the editors, and the reviewers. Any product that may be evaluated in this article, or claim that may be made by its manufacturer, is not guaranteed or endorsed by the publisher.

Copyright $\odot 2022$ Yu, Li, Zhang, Guo, Cao, Guo, Wu, Li and Pang. This is an openaccess article distributed under the terms of the Creative Commons Attribution License (CC BY). The use, distribution or reproduction in other forums is permitted, provided the original author(s) and the copyright owner(s) are credited and that the original publication in this journal is cited, in accordance with accepted academic practice. No use, distribution or reproduction is permitted which does not comply with these terms. 